\title{
Graph Design for Secure Multiparty Computation over Non-Abelian Groups
}

\author{
Xiaoming $\operatorname{Sun}^{1}$, Andrew Chi-Chih $\mathrm{Yao}^{1}$, and Christophe Tartary ${ }^{1,2}$ \\ ${ }^{1}$ Institute for Theoretical Computer Science \\ Tsinghua University \\ Beijing, 100084 \\ People's Republic of China \\ 2 Division of Mathematical Sciences \\ School of Physical and Mathematical Sciences \\ Nanyang Technological University \\ Singapore \\ \{xiaomings, andrewcyao\} atsinghua. edu.cn, \\ ctartary@ntu.edu.sg
}

\begin{abstract}
Recently, Desmedt et al. studied the problem of achieving secure $n$ party computation over non-Abelian groups. They considered the passive adversary model and they assumed that the parties were only allowed to perform black-box operations over the finite group $G$. They showed three results for the $n$-product function $f_{G}\left(x_{1}, \ldots, x_{n}\right):=x_{1} \cdot x_{2} \cdot \ldots \cdot x_{n}$, where the input of party $P_{i}$ is $x_{i} \in G$ for $i \in\{1, \ldots, n\}$. First, if $t \geq\left\lceil\frac{n}{2}\right\rceil$ then it is impossible to have a $t$-private protocol computing $f_{G}$. Second, they demonstrated that one could $t$ privately compute $f_{G}$ for any $t \leq\left\lceil\frac{n}{2}\right\rceil-1$ in exponential communication cost. Third, they constructed a randomized algorithm with $O\left(n t^{2}\right)$ communication complexity for any $t<\frac{n}{2.948}$.

In this paper, we extend these results in two directions. First, we use percolation theory to show that for any fixed $\epsilon>0$, one can design a randomized algorithm for any $t \leq \frac{n}{2+\epsilon}$ using $O\left(n^{3}\right)$ communication complexity, thus nearly matching the known upper bound $\left\lceil\frac{n}{2}\right\rceil-1$. This is the first time that percolation theory is used for multiparty computation. Second, we exhibit a deterministic construction having polynomial communication cost for any $t=O\left(n^{1-\epsilon}\right)$ (again for any fixed $\epsilon>0$ ). Our results extend to the more general function $\widetilde{f}_{G}\left(x_{1}, \ldots, x_{m}\right):=x_{1} \cdot x_{2} \cdot \ldots \cdot x_{m}$ where $m \geq n$ and each of the $n$ parties holds one or more input values.
\end{abstract}

Keywords: Multiparty Computation, Passive Adversary, Non-Abelian Groups, Graph Coloring, Percolation Theory.

\section{Introduction}

In multiparty computation, a set of $n$ parties $\left\{P_{1}, \ldots, P_{n}\right\}$ want to compute a function of some secret inputs held locally by these participants. Since its introduction by Yao [19], multiparty computation has been extensively studied. Most multiparty computation protocols rely on algebraic structures which are at least Abelian groups [14] as in 
[1, 3, 4, 8, 10, 11, 12] for instance. The usefulness of Abelian groups in cryptography is not restricted to multiparty computation as numerous cryptographic primitives are developed over such groups [6, 7, 17]. However, the construction of efficient quantum algorithms to solve the discrete logarithm problem as well as the factoring problem prevent the use of many of these primitives over those machines [18]. Since quantum algorithms seem to be less efficient over non-Abelian groups, there is increasingly a need for developing cryptographic constructions over such mathematical structures. The reader may be aware of the existence of public key cryptosystems for such groups [15, 16].

Recently, Desmedt et al. studied the problem of designing secure $n$-party protocol over non commutative finite groups for the passive (or semi-honest) adversary model [5]. Their goal is to guarantee unconditional security simply using a black-box representation of the finite non-Abelian group $(G, \cdot)$. This assumption means that the $n$ parties can only perform three operations in $(G, \cdot)$ : the group operation $((x, y) \mapsto x \cdot y)$, the group inversion $\left(x \mapsto x^{-1}\right)$ and the uniformly distributed group sampling $\left(x \in_{R} G\right)$.

Desmedt et al. focused on the existence and the design of $t$-private protocols for the $n$ product function $f_{G}\left(x_{1}, \ldots, x_{n}\right):=x_{1} \cdot \ldots \cdot x_{n}$ where the input of party $P_{i}$ is $x_{i} \in G$ for $i \in\{1, \ldots, n\}$. In such a protocol, no colluding sets $\mathcal{C}$ of at most $t$ participants learn anything about the data hold by any of the remaining members $\left\{P_{1}, \ldots, P_{n}\right\} \backslash \mathcal{C}$. Desmedt et al. obtained three important results. First, if $t \geq\left\lceil\frac{n}{2}\right\rceil$ (dishonest majority) then it is impossible to construct a $t$-private protocol to compute $f_{G}$. Second, if $t<\left\lceil\frac{n}{2}\right\rceil$ then one can always design a deterministic $t$-private protocol computing $f_{G}$ with an exponential communication complexity of $O\left(n\left(\begin{array}{c}2 t+1 \\ t\end{array}\right)^{2}\right)$ group elements. Third, they built a probabilistic $t$-private protocol computing $f_{G}$ with a polynomial communication complexity of $O\left(n t^{2}\right)$ group elements when $t<\frac{n}{2.948}$.

That work leads to two important questions. First, we would like to know if it is possible to construct a $t$-private protocol for values of $t \in\left[\frac{n}{2.948},\left\lceil\frac{n}{2}\right\rceil-1\right]$ with polynomial communication complexity. Second, Desmedt et al.'s construction shows that one can $t$-privately compute $f_{G}$ with polynomial communication cost for any $t=O(\log n)$. A natural issue is to determine the existence and to construct a deterministic $t$-private protocol with polynomial communication complexity for other values $t$ (ideally, up to the threshold $\left\lceil\frac{n}{2}\right\rceil-1$ ).

In this article, we give a positive answer to these two questions. First, we demonstrate that the random coloring approach and the graph construction by Desmedt et al. can be used to guarantee $t$-privacy for any $t<\frac{n}{2+\epsilon}$ (for any fixed $\epsilon>0$ ). The communication complexity of our construction is $O\left(n^{3}\right)$ group elements. This result is obtained using percolation theory. To the best of our knowledge, this is the first use of this theory in the context of multiparty computation. Second, we provide a deterministic construction for any $t=O\left(n^{1-\epsilon}\right)$. This scheme has polynomial communication complexity as well.

This paper is organized as follows. In the next section, we will recall the different reductions performed in [5] to solve the $t$-privacy issue over non-Abelian groups. In Sect. 3, we present our randomized construction achieving $t$-privacy for any value $t \leq$ $\frac{n}{2+\epsilon}$ which is closed to the theoretical bound $\left\lceil\frac{n}{2}\right\rceil-1$. In Sect. 4 , we show how to construct deterministic $t$-private protocols having polynomial communication cost for any $t=O\left(n^{1-\epsilon}\right)$. In the last section, we conclude our paper with some remaining open problems for multiparty computation over non-Abelian black-box groups. 


\section{Achieving Secure Computation over Non-Abelian Groups}

In this section, we present some of the results and constructions developed by Desmedt et al. which are necessary to understand our improvements from Sect. 3 and Sect. 4 First, we recall the definition of secure multiparty computation in the passive, computationally unbounded attack model, restricted to deterministic symmetric functionalities and perfect emulation as in [5].

We denote $[n]$ the set of integers $\{1, \ldots, n\},\{0,1\}^{*}$ the set of all finite binary strings and $|A|$ the cardinality of the set $A$.

Definition 1. We denote $f:\left(\{0,1\}^{*}\right)^{n} \mapsto\{0,1\}^{*}$ an $n$-input and single-output function. Let $\prod$ be a n-party protocol for computing $f$. We denote the n-party input sequence by $\mathbf{x}=\left(x_{1}, \ldots, x_{n}\right)$, the joint protocol view of parties in subset $I \subset[n]$ by $\operatorname{VIEW}_{I}^{\prod}(\mathbf{x})$, and the protocol output by $\operatorname{OUT}^{\Pi}(\mathbf{x})$. For $0<t<n$, we say that $\prod$ is a t-private protocol for computing $f$ if there exists a probabilistic polynomial-time algorithm $S$, such that, for every $I \subset[n]$ with $|I| \leq t$ and every $\mathbf{x} \in\left(\{0,1\}^{*}\right)^{n}$, the random variables

$$
\left\langle S\left(I, \mathbf{x}_{I}, f(\mathbf{x})\right), f(\mathbf{x})\right\rangle \text { and }\left\langle\mathbf{V I E W} I_{I}^{\prod}(\mathbf{x}), \mathbf{O U T}^{\Pi}(\mathbf{x})\right\rangle
$$

are identically distributed, where $\mathbf{x}_{I}$ denotes the projection of the $n$-ary sequence $\mathbf{x}$ on the coordinates in $I$.

In the remaining of this paper, we assume that party $P_{i}$ has a personal input $x_{i} \in G$ (for $i \in[n]$ ) and the function to be computed is the $n$-party product $f_{G}\left(x_{1}, \ldots, x_{n}\right):=$ $x_{1} \cdot \ldots \cdot x_{n}$.

Desmedt et al. first reduced the problem of constructing a $t$-private $n$-party protocol for $f_{G}$ to the problem of constructing a symmetric (strong) t-private protocol $\prod^{\prime}$ (see [5] for a detailed definition of symmetric privacy) to compute the shared 2-product function $f_{G}^{\prime}(x, y):=x \cdot y$ where the inputs $x$ and $y$ are shared amongst the $n$ parties. They demonstrated that iterating $(n-1)$ times the protocol $\prod^{\prime}$ would give a $t$-private protocol to compute $f_{G}$.

The second reduction occurring in [5] consists of constructing a $t$-private $n$-party shared 2-product protocol $\prod^{\prime}$ from a suitable coloring over particular directed graphs. We will detail the important steps of this reduction as they will serve the understanding of our own constructions.

Definition 2 ([5]). We call graph $\mathcal{G}$ an admissible Planar Directed Acyclic Graph (PDAG) with share parameter $\ell$ and size parameter $m(\geq \ell)$ if it has the following properties:

- The nodes of $\mathcal{G}$ are drawn on a square $m \times m$ grid of points (each node of $\mathcal{G}$ is located at a grid point but some grid points may not be occupied by nodes). The rows of the grid are indexed from top to bottom and the columns from left to right by the integers $1,2, \ldots, m$. A node of $\mathcal{G}$ at row $i$ and column $j$ is said to have index $(i, j) . \mathcal{G}$ has $2 \ell$ input nodes on the top row, and $\ell$ output nodes on the bottom row.

- The incoming edges of a node on row $i$ only come from nodes on row $i-1$, and outgoing edges of a node on row $i$ only go to nodes on row $i+1$. 
- For each row $i$ and column $j$, let $\eta_{1}^{(i, j)}<\cdots<\eta_{q^{(i, j)}}^{(i, j)}$ denote the ordered column indices of the $q(i, j)>0$ nodes on level $i+1$ which are connected to node $(i, j)$ by an edge. Then, for each $j \in[m-1]$, we have:

$$
\eta_{q(i, j)}^{(i, j)} \leq \eta_{1}^{(i, j+1)}
$$

which means that the rightmost node on level $i+1$ connected to node $(i, j)$ is to the left of (or equal to) the leftmost node on level $i+1$ connected to node $(i, j+1)$.

An admissible PDAG has $2 \ell$ input nodes. The first $\ell$ ones $($ i.e. $(1,1), \ldots,(1, \ell))$ represent the $x$-input nodes while the remaining ones represent the $y$-input nodes. Let $C:[m] \times[m] \mapsto[n]$ be a $n$-coloring function that associates to each node $(i, j)$ of $\mathcal{G}$ a color $C(i, j)$ chosen from a set of $n$ possible colors. The following notion will be used to express the property we expect the graph coloring to have in order to build $\prod^{\prime}$.

Definition $3([\mathbf{5}])$. We say that $C:[m] \times[m] \mapsto[n]$ is a $t$-reliable $n$-coloring for the admissible PDAG $\mathcal{G}$ (with share parameter $\ell$ and size parameter $m$ ) if for each $t$-color subset $I \subset[n]$, there exist $j^{*} \in[\ell]$ and $j_{y}^{*} \in[\ell]$ such that:

- There exists a path $\mathrm{PATH}_{x}$ in $\mathcal{G}$ from the $j^{*}$ th $x$-input node to the $j^{*}$ th output node, such that none of the path node colors are in subset I (it is called an I-avoiding path), and

- There exists an I-avoiding path $\mathrm{PATH}_{y}$ in $\mathcal{G}$ from the $j_{y}^{*}$ th $y$-input node to the $j^{*}$ th output node.

If $j_{y}^{*}=j^{*}$ for all $I$, we say that $C$ is a symmetric $t$-reliable $n$-coloring.

Important Remark: Even if the graph $\mathcal{G}$ is directed, it is regarded as non-directed when building the $I$-avoiding paths in Definition 3

Desmedt et al. built a protocol $\prod^{\prime}(\mathcal{G}, C)$ taking as input a graph $\mathcal{G}$ and a $n$ coloring $C$. We do not detail this protocol in our paper as its internal design does not have any influence in our work. The reader can find it in [5]. However, in order to ease the understanding of our work, we recall the relation between multiparty protocols over a non-Abelian group $G$ and coloring of admissible PDAGs as it appear in [5].

The $n$ participants $\left\{P_{1}, \ldots, P_{n}\right\}$ are identified by the $n$ colors of the admissible PDAG $\mathcal{G}$. The input/output nodes of the graph $\mathcal{G}$ are labeled by the input/output elements of the group $G$. Each edge represents a group element sent from one participant to another one. Each internal node contains an intermediate value of the protocol. Those values are computed, at each node $\mathcal{N}$ of $\mathcal{G}$, as the group operation between the elements along all the incoming edges of $\mathcal{N}$ from the leftmost one to the rightmost one. This intermediate value is then redistributed along all the outgoing edges of $\mathcal{N}$ using the following $O_{\mathcal{N}}$-of- $O_{\mathcal{N}}$ secret sharing where $O_{\mathcal{N}}$ represents the number of outgoing edges of node $\mathcal{N}$.

Proposition 1 ([5]). Let $g$ be an element of the non-Abelian group G. Denote $\lambda$ and $\mu$ two integers where $\mu \in[\lambda]$. We create a $\lambda$-of- $\lambda$ sharing $\left(s_{g}(1), \ldots, s_{g}(\lambda)\right)$ of $g$ by picking the $\lambda-1$ shares $\left\{s_{g}(\xi)\right\}_{\xi \in[\lambda] \backslash\{\mu\}}$ uniformly and independently at random from $G$, and computing $s_{g}(\mu)$ to be the unique element of $G$ such that:

$$
g=s_{g}(1) \cdot s_{g}(2) \cdot \ldots \cdot s_{g}(\lambda)
$$


Then, the distribution of the shares $\left(s_{g}(1), \ldots, s_{g}(\lambda)\right)$ is independent of $\mu$.

We recall the following important result:

Theorem 1 ([5]). If $\mathcal{G}$ is an admissible PDAG and $C$ is a symmetric t-reliable $n$ coloring for $\mathcal{G}$ then $\prod^{\prime}(\mathcal{G}, C)$ achieves symmetric strong t-privacy.

The last reduction is related to the admissible PDAG. Desmedt et al. only consider admissible PDAGs as defined below and represented in Fig. 1

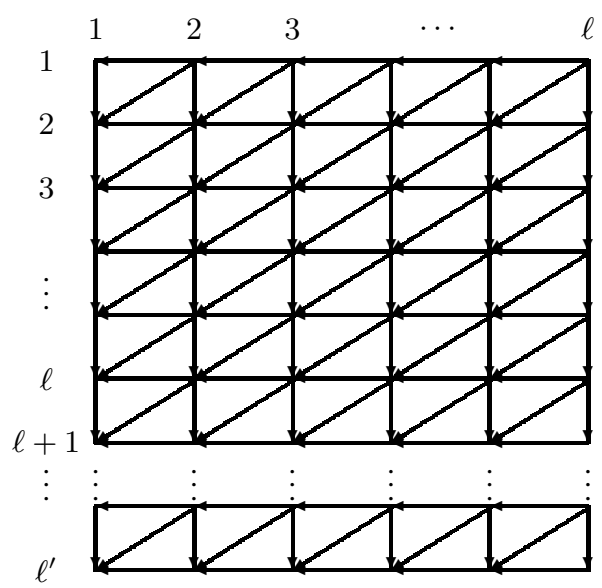

Fig. 1. The admissible PDAG $\mathcal{G}_{\text {tri }}\left(\ell^{\prime}, \ell\right)$

Definition 4 ([5]). The admissible PDAG $\mathcal{G}_{\text {tri }}\left(\ell^{\prime}, \ell\right)$ is a $\ell^{\prime} \times \ell$ directed grid such that:

- [horizontal edges] for $i \in\left[\ell^{\prime}\right]$ and for $j \in[\ell-1]$, there is a directed edge from node $(i, j+1)$ to $(i, j)$,

- [vertical edges] for $i \in\left[\ell^{\prime}-1\right]$ and for $j \in[\ell]$, there is a directed edge from node $(i, j)$ to node $(i+1, j)$,

- [diagonal edges] for $i \in\left[\ell^{\prime}-1\right]$ and for $j \in\{2, \ldots, \ell\}$, there is a directed edge from node $(i, j)$ to node $(i+1, j-1)$.

According to Definition 2] an admissible PDAG has $2 \ell$ input nodes and no horizontal edges. Desmedt et al. indicated that the $y$-input nodes could be arranged along a column on $\mathcal{G}_{\text {tri }}\left(\ell^{\prime}, \ell\right)$ instead of being along the same row as the $x$-input nodes. They also explained that $\mathcal{G}_{\text {tri }}\left(\ell^{\prime}, \ell\right)$ could also be drawn according the requirements of Definition 2 By rotating $\mathcal{G}_{\text {tri }}\left(\ell^{\prime}, \ell\right)$ by 45 degrees anticlockwise, the $x$-input nodes and $y$-input nodes of $\mathcal{G}_{\text {tri }}\left(\ell^{\prime}, \ell\right)$ are now on the same row and the horizontal edges of $\mathcal{G}_{\text {tri }}\left(\ell^{\prime}, \ell\right)$ have become diagonal edges which satisfies Definition 2 .

A priori, $\mathcal{G}_{\text {tri }}\left(\ell^{\prime}, \ell\right)$ is a rectangular grid. In [5], Desmedt et al. considered square grids $\mathcal{G}_{\text {tri }}(\ell, \ell)$ for which they introduced the following notion.

Definition 5 ([5]). We say that $C:[\ell] \times[\ell] \mapsto[n]$ is a weakly t-reliable $n$-coloring for $\mathcal{G}_{\text {tri }}(\ell, \ell)$ if for each $t$-color subset $I \subset[n]$ : 
- There exists an I-avoiding path $\mathcal{P}_{x}$ in $\mathcal{G}_{\text {tri }}(\ell, \ell)$ from a node on the top row to a node on the bottom row. Such a path is called an I-avoiding top-bottom path.

- There exists an I-avoiding path $\mathcal{P}_{y}$ in $\mathcal{G}_{\text {tri }}(\ell, \ell)$ from a node on the rightmost column to a node on the leftmost column. Such a path is called an I-avoiding right-left path.

As said in [5], the admissible PDAG requirements (Definition 2) are still satisfied if we remove from $\mathcal{G}_{\text {tri }}$ some 'positive slope' diagonal edges and add some 'negative slope' diagonal edges (connecting a node $(i, j)$ to node $(i+1, j+1)$, for some $i \in\left[\ell^{\prime}-1\right]$ and $j \in[\ell-1]$ ). Such a generalized admissible PDAG is denoted $\mathcal{G}_{\text {gtri }}$.

Lemma 1 ([5]). Let $C:[\ell] \times[\ell] \mapsto[n]$ be a weakly t-reliable $n$-coloring for square admissible PDAG $\mathcal{G}_{\text {tri }}(\ell, \ell)$. Then, we can construct a t-reliable $n$-coloring for a rectangular admissible PDAG $\mathcal{G}_{\text {gtri }}(2 \ell-1, \ell)$.

Thus, Desmedt et al. have demonstrated that it was sufficient to get a weakly $t$-reliable $n$ coloring for some $\mathcal{G}_{\text {tri }}(\ell, \ell)$ in order to construct a $t$-private protocol for computing the $n$-product $f_{G}$. The cost communication cost of this protocol is $(n-1)$ times the number of edges of $\mathcal{G}_{\text {gtri }}(2 \ell-1, \ell)$. Since that grid is obtained from $\mathcal{G}_{\text {tri }}(\ell, \ell)$ using a mirror, the communication cost of the whole protocol is $O\left(n \ell^{2}\right)$ group elements. The constructions that we propose in this paper are colorings of some grids $\mathcal{G}_{\text {tri }}(\ell, \ell)$.

\section{A Randomized Construction Achieving Maximal Privacy}

In this section, we present a randomized construction ensuring the $t$-privacy of the computation of $f_{G}$ up to $\frac{n}{2+\epsilon}$. Our scheme has a linear share parameter $\ell=O(n)$.

We use the same random coloring $C_{\text {rand }}$ for the grid $\mathcal{G}_{\text {tri }}(\ell, \ell)$ as in [5]. However, our analysis is based on percolation theory while Desmedt et al. used a counting-based argument. We first introduce the following definition which is illustrated in Fig. 2,

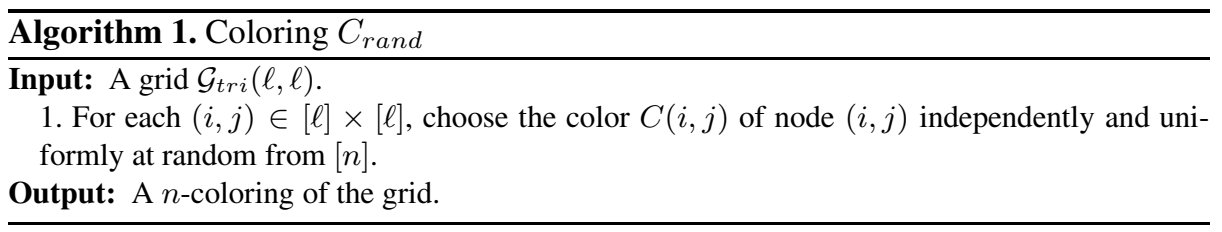

Definition 6. The triangular lattice of depth $\ell$ denoted $\mathcal{T}(\ell)$ is a directed graph drawn over a $\ell \times(3 \ell-2)$ grid such that:

- [horizontal edges] for $i \in[\ell]$ and for $j \in[\ell-1]$, there is a directed edge from node $(i, i+2 j)$ to $(i, i+2(j-1))$,

- [right downwards edges] for $i \in[\ell-1]$ and for $j \in\{0, \ldots, \ell-1\}$, there is a directed edge from node $(i, i+2 j)$ to node $(i+1, i+2 j+1)$,

- [left downwards edges] for $i \in[\ell-1]$ and for $j \in[\ell-1]$, there is a directed edge from node $(i, i+2 j)$ to node $(i+1, i+2 j-1)$. 


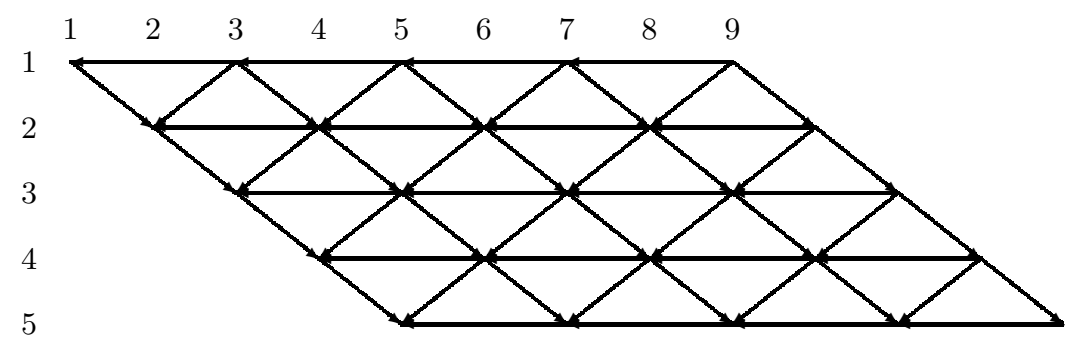

Fig. 2. The triangle $\mathcal{T}(5)$

Proposition 2. For any positive integer $\ell$, we have a graph isomorphism between $\mathcal{G}_{\text {tri }}(\ell, \ell)$ and $\mathcal{T}(\ell)$.

Proof. Consider the mapping:

$$
\begin{aligned}
\mathcal{G}_{\text {tri }}(\ell, \ell) & \longrightarrow \mathcal{T}(\ell) \\
(i, j) & \longmapsto(i, i+2(j-1))
\end{aligned}
$$

It is easy to see that the nodes of the two graphs are in bijective correspondence while the direction of each edge is maintained.

Theorem 2. For any $\epsilon>0$, there exists a constant $c_{\epsilon}$ such that if $\leq \frac{n}{2+\epsilon}$ and $\ell \geq c_{\epsilon} n$, then there exists a weakly $t$-reliable $n$-coloring for $\mathcal{G}_{\text {tri }}(\ell, \ell)$.

Proof. We prove that the coloring $C_{\text {rand }}$ will work with high probability. Let $t_{\epsilon}=$ $\left\lfloor\frac{n}{2+\epsilon}\right\rfloor$ where $\lfloor\cdot\rfloor$ denotes the floor function. Instead of considering the probability that $C_{\text {rand }}$ is a weakly $t_{\epsilon}$-reliable $n$-coloring for $\mathcal{G}_{\text {tri }}(\ell, \ell)$, we study the complementary event. A suitable value for $\ell$ will be given at the end of this demonstration.

The coloring $C_{\text {rand }}$ is called bad if there exists a color set $I \subset[n]$ with $|I|=t_{\epsilon}$, such that either there are no $I$-avoiding top-bottom paths or there are no $I$-avoiding right-left paths. By the union bound, we obtain the following upper bound on $\operatorname{Pr}\left(C_{\text {rand }}\right.$ is bad $)$ :

$2 \operatorname{Pr}\left(\exists I \subset[n],|I|=t_{\epsilon}\right.$, there are no $I$-avoiding top-bottom paths in $\left.\mathcal{G}_{\text {tri }}(\ell, \ell)\right)$

$\leq 2 \sum_{I \subset[n],|I|=t_{\epsilon}} \operatorname{Pr}\left(\right.$ there are no $I$-avoiding top-bottom paths in $\left.\mathcal{G}_{\text {tri }}(\ell, \ell)\right)$.

The factor 2 in (1) comes from the fact the top-bottom probability is equal to the rightleft probability due to the symmetry of the grid $\mathcal{G}_{\text {tri }}(\ell, \ell)$ and the coloring $C_{\text {rand }}$.

Next, we demonstrate that for a fixed color set $I \subset[n]$ with $|I|=t_{\epsilon}$, the probability that there are no $I$-avoiding top-bottom paths in $C_{\text {rand }}$ is exponentially small. Let us fix the color set $I$. We call a vertex closed if its color belongs to $I$. Otherwise, the vertex is called open. The random coloring $C_{\text {rand }}$ of each vertex is equivalent to open it independently and randomly with probability $p:=1-\frac{t_{\epsilon}}{n}$. An $I$-avoiding path is simply an open path. Therefore, we get:

$\operatorname{Pr}\left(\right.$ there are no $I$-avoiding top-bottom paths in $\left.\mathcal{G}_{\text {tri }}(\ell, \ell)\right)$

$=\operatorname{Pr}_{p}\left(\right.$ there are no open top-bottom paths in $\left.\mathcal{G}_{\text {tri }}(\ell, \ell)\right)$

$=1-\operatorname{Pr}_{p}\left(\right.$ there is an open top-bottom path in $\left.\mathcal{G}_{\text {tri }}(\ell, \ell)\right)$ 
We have the following result.

Lemma 2 ([2]). The triangular lattice $\mathcal{T}(\ell)$ has the following property:

$$
\begin{gathered}
\left.\operatorname{Pr}_{p} \text { (there is an open top-bottom path in } \mathcal{T}(\ell)\right) \\
+ \\
\operatorname{Pr}_{p}(\text { there is a closed right-left path in } \mathcal{T}(\ell)) \\
=1
\end{gathered}
$$

When we combine Lemma 2 Proposition 2 and (2), we obtain the following:

$\operatorname{Pr}\left(\right.$ there is no $I$-avoiding top-bottom path in $\left.\mathcal{G}_{\text {tri }}(\ell, \ell)\right)$

$$
\begin{gathered}
= \\
\operatorname{Pr}_{p}(\text { there is a closed right-left path in } \mathcal{T}(\ell)) \\
= \\
\operatorname{Pr}_{1-p}(\text { there is an open right-left path in } \mathcal{T}(\ell))
\end{gathered}
$$

In (3), $\operatorname{Pr}_{1-p}(\cdot)$ means that we open each vertex with probability $1-p$. We have the following result from percolation theory.

Lemma 3 ([13]). Let $T$ be the triangular lattice in the plane. Then, the critical probability of site percolation $p_{c}^{s}(T)$ is equal to $\frac{1}{2}$.

When the open probability is less than the critical probability, the percolation has the following properties (see for example Chapter 4, Theorem 9 in [2]).

Lemma 4 ([9]). If $p<p_{c}^{s}(T)$, then there is a constant $c=c(p)$,

$$
\operatorname{Pr}_{p}(0 \stackrel{n}{\longrightarrow})<e^{-c n} \text {. }
$$

where $\{x \stackrel{n}{\longrightarrow}\}$ is the event that there is an open path from $x$ to a point in $S_{n}(x)$ with $S_{n}(x):=\{y: d(x, y)=n\}$ and $d(x, y)$ denotes the distance between $x$ and $y$.

Remark: The value 0 from Lemma 4 represent the zero element of $\mathbb{Z} \times \mathbb{Z}$ when the graph is represented as a lattice over that set. In the case of the triangular lattice depicted as Fig. 2, the value 0 can be identified to the node $(1,1)$.

In our case, we have: $1-p=\frac{t_{\epsilon}}{n} \leq \frac{1}{2+\epsilon}<p_{c}^{s}(T)$. Using Lemma4, we get:

$$
\operatorname{Pr}_{1-p}(\text { there is an open right-left path in } \mathcal{T}(\ell)) \leq \ell \operatorname{Pr}_{1-p}(0 \stackrel{\ell-1}{\longrightarrow}) \leq \ell e^{-c(\ell-1)}
$$

The first inequality is due to the fact that any right-left path has length at least $(\ell-1)$ in $\mathcal{T}(\ell)$. Combining (1)-(4), we obtain:

$$
\operatorname{Pr}\left(C_{\text {rand }} \text { is bad }\right) \leq 2\left(\begin{array}{c}
n \\
t_{\epsilon}
\end{array}\right) \ell e^{-c(\ell-1)}
$$

Thus, if we choose $\ell:=c_{\epsilon} n$ for some large enough constant $c_{\epsilon}$, we have:

$$
\operatorname{Pr}\left(C_{\text {rand }} \text { is bad }\right) \leq \frac{1}{2^{n}}
$$

which guarantees the fact that $C_{\text {rand }}$ is a weakly $t_{\epsilon}$-reliable $n$-coloring for $\mathcal{G}_{\text {tri }}(\ell, \ell)$ with overwhelming probability in $n$.

Corollary 1. There exists a black box $t_{\epsilon}$-private protocol for $f_{G}$ with communication complexity $O\left(n^{3}\right)$ group elements where $t_{\epsilon}=\left\lfloor\frac{n}{2+\epsilon}\right\rfloor$. Moreover, for any $\delta>0$, we 
can construct a probabilistic algorithm, with run-time polynomial in $n$ and $\log \left(\delta^{-1}\right)$, which outputs a protocol $\prod$ for $f_{G}$ such that the communication complexity of $\prod$ is $O\left(n^{3} \log ^{2}\left(\delta^{-1}\right)\right)$ group elements and the probability that $\prod$ is not $t_{\epsilon}$-private is at most $\delta$.

Proof. The existence of the protocol is a direct consequence of Theorem 2 as well as the different reductions exposed in Sect. 2. As our construction requires $\ell=O(n)$, we deduce that the communication cost of the protocol computing $f_{G}$ is $O\left(n^{3}\right)$. The justification of the running time of the algorithm and the probability of failure $\delta$ is identical to what is done in [5].

We showed that it was possible to build a randomized algorithm to achieve $\left\lfloor\frac{n}{2+\epsilon}\right\rfloor$ private computation of $f_{G}$ using $O\left(n^{3}\right)$ group elements. Even if the probability of failure of our previous construction is small, we would like to remove the randomized restriction so that we can get a (deterministic) protocol which is always guaranteed to succeed. In [5], Desmedt et al. only provided deterministic protocols to compute $f_{G}$ in polynomial communication cost when $t=O(\log n)$. In the next section, we present a deterministic construction for any $t=O\left(n^{1-\epsilon}\right)$ where $\epsilon$ is any positive constant. Our construction requires polynomial communication complexity as well.

\section{A Deterministic Construction for Secure Computation}

In this section, we show how to build a deterministic $t$-private protocol to compute $f_{G}$ with polynomial complexity cost for any $t=O\left(n^{1-\epsilon}\right)$. First, we will focus on particular pairs $(t, n)$. Second, we generalize our result to any $(t, n)$ with $t=O\left(n^{1-\epsilon}\right)$.

We recursively construct our admissible PDAG $\mathcal{G}_{\text {rec }}$ and its coloring $C_{r e c}$. Let $d \in$ $\mathbb{N} \backslash\{0,1\}$ be a constant. Denote $\mathcal{B}_{d}$ the binomial coefficient $\left(\begin{array}{c}2 d-1 \\ d-1\end{array}\right)$.

Theorem 3. For any positive integer $k$, there is a weakly $t_{k}$-reliable $n_{k}$-coloring $C_{\text {rec }}\left(\ell_{k}\right)$ for the square admissible PDAG $\mathcal{G}_{\text {rec }}\left(\ell_{k}\right)$, where the parameters are: $t_{k}:=d^{k}-1, n_{k}:=(2 d-1)^{k}$ and $\ell_{k}=\mathcal{B}_{d}^{k}\left(\mathcal{B}_{d}+1\right)^{k-1}$.

Proof. We prove the theorem by induction on $k$.

$k=1$ : We have $t_{1}=d-1, n_{1}=2 d-1$ and $\ell_{1}=\mathcal{B}_{d}$. We set $\mathcal{G}_{r e c}\left(\ell_{1}\right):=\mathcal{G}_{\text {tri }}\left(\ell_{1}, \ell_{1}\right)$. We define $C_{r e c}\left(\ell_{1}\right)$ as being the combinatorial coloring $C_{\text {comb }}$ designed in [5] and recalled as Algorithm 2.

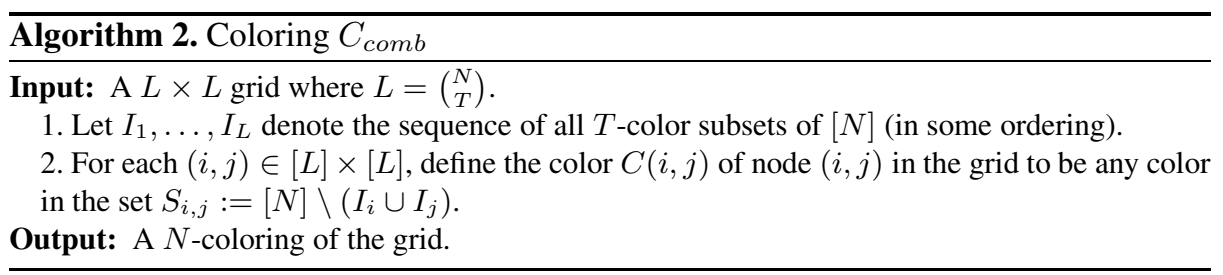


Desmedt et al. noticed that, even if we removed the diagonal edges from $\mathcal{G}_{\text {tri }}\left(\ell_{1}, \ell_{1}\right)$, we still had the existence of $I$-avoiding top-bottom and right-left paths. Thus, we assume that $\mathcal{G}_{\text {rec }}\left(\ell_{1}\right)$ has no such edges so that $\mathcal{G}_{\text {rec }}\left(\ell_{1}\right)$ is a square grid the side length of which is $\ell_{1}$ nodes. $\mathcal{G}_{\text {rec }}\left(\ell_{1}\right)$ is an admissible PDAG.

$k \geq 1$ : Suppose we already have the construction and coloring for $k$, we recursively construct $\mathcal{G}_{\text {rec }}\left(\ell_{k+1}\right)$ from $\mathcal{G}_{\text {rec }}\left(\ell_{k}\right)$.

We first build the block grid $B$ by copying $\left(\mathcal{B}_{d}+1\right) \times\left(\mathcal{B}_{d}+1\right)$ times $\mathcal{G}_{\text {rec }}\left(\ell_{1}\right)$. The connections between two copies of $\mathcal{G}_{\text {rec }}\left(\ell_{1}\right)$ are as follows. Horizontally, we draw a directed edge from node $(i, 1)$ in the right-hand side copy to node $\left(i, \ell_{1}\right)$ in the lefthand side copy for $i \in\left[\ell_{1}\right]$ (i.e. we horizontally connect nodes at the same level). Vertically, we draw a directed edge from node $\left(\ell_{1}, j\right)$ in the top side copy to node $(1, j)$ in the bottom side copy for $j \in\left[\ell_{1}\right]$ (i.e. we vertically connect nodes at the same level).

The block $B$ is a $\left(\mathcal{B}_{d}\left(\mathcal{B}_{d}+1\right)\right) \times\left(\mathcal{B}_{d}\left(\mathcal{B}_{d}+1\right)\right)$ grid. It has the following property the proof of which can be found in Appendix $\mathrm{A}$.

Proposition 3. The block grid $B$ admits a $(2 d-1)$-coloring (just use the same $C_{\text {comb }}$ for each copy of $\left.\mathcal{G}_{\text {rec }}\left(\ell_{1}\right)\right)$, such that for any $(d-1)$-color subset $I \subset[2 d-1]$, there are $\mathcal{B}_{d}+1$ horizontal (vertical) $I$-avoiding straight lines in $B$.

Now, we construct $\mathcal{G}_{\text {rec }}\left(\ell_{k+1}\right)$ and its coloring $C_{\text {rec }}\left(\ell_{k+1}\right)$ as follows. We replace each node in $\mathcal{G}_{\text {rec }}\left(\ell_{k}\right)$ by a copy of $B$. If the node of $\mathcal{G}_{\text {rec }}\left(\ell_{k}\right)$ was colored by the color $c \in\left[n_{k}\right]$, then we color $B$ with the set of colors $\{(2 d-1)(c-1)+1,(2 d-1)(c-1)+$ $2, \ldots,(2 d-1) c\}$, using $C_{c o m b}$. All the edges within each copy of $B$ remain identical in $\mathcal{G}_{\text {rec }}\left(\ell_{k+1}\right)$.

Now, we show how to connect two copies of $B$. We first focus on vertical connections. Consider an edge in $\mathcal{G}_{\text {rec }}\left(\ell_{k}\right)$ from a node in the $i$-th row to another node in the $(i+1)$-th row. Since these two nodes have been replaced by two copies of $B$, we denote the nodes on the top copy (i.e. those corresponding to the nodes of the $i$-th row in $\left.\mathcal{G}_{r e c}\left(\ell_{k}\right)\right)$ as $v_{1,1}, \ldots, v_{1, \mathcal{B}_{d}}, v_{2,1}, \ldots, v_{\mathcal{B}_{d}+1, \mathcal{B}_{d}}$ and the nodes on the bottom copy as $w_{1,1}, \ldots, w_{1, \mathcal{B}_{d}}, w_{2,1}, \ldots, w_{\mathcal{B}_{d}+1, \mathcal{B}_{d}}$.

For each $(i, j) \in\left[\mathcal{B}_{d}\right] \times\left[\mathcal{B}_{d}\right]$, we add a directed edge $\left(v_{i, j}, w_{i, j+i-1}\right)$ in $\mathcal{G}_{r e c}\left(\ell_{k+1}\right)$. If the index $(j+i-1)$ is greater than $\mathcal{B}_{d}, w_{i, j+i-1}$ is the node $w_{i+1, j+i-1-\mathcal{B}_{d}}$. Figure 3 gives the example for $d=2$. The connection process works similarly for two consecutive columns where we replace each horizontal edge from $\mathcal{G}_{r e c}\left(\ell_{k}\right)$ by $\mathcal{B}_{d}^{2}$ different edges in $\mathcal{G}_{r e c}\left(\ell_{k+1}\right)$.

It is clear that the number of nodes on each side of the square $\mathcal{G}_{r e c}\left(\ell_{k+1}\right)$ is:

$$
\ell_{k+1}=\mathcal{B}_{d}\left(\mathcal{B}_{d}+1\right) \cdot \ell_{k}=\mathcal{B}_{d}^{k+1}\left(\mathcal{B}_{d}+1\right)^{k}
$$

and the number of colors used in $C_{r e c}\left(\ell_{k+1}\right)$ is $n_{k+1}=(2 d-1) \cdot n_{k}=(2 d-1)^{k+1}$. The grid $\mathcal{G}_{\text {rec }}\left(\ell_{k+1}\right)$ obtained by this recursive process is also an admissible PDAG due to the horizontal/vertical connection processes between two copies of $B$ (as well as two copies of $\mathcal{G}_{\text {rec }}\left(\ell_{1}\right)$ inside $B$ ).

The last point to prove is that for any $t_{k+1}$-color subset $I \subset\left[n_{k+1}\right]$, there is an $I$-avoiding top-bottom (and right-left) path in $\mathcal{G}_{r e c}\left(\ell_{k+1}\right)$. We only prove the existence 


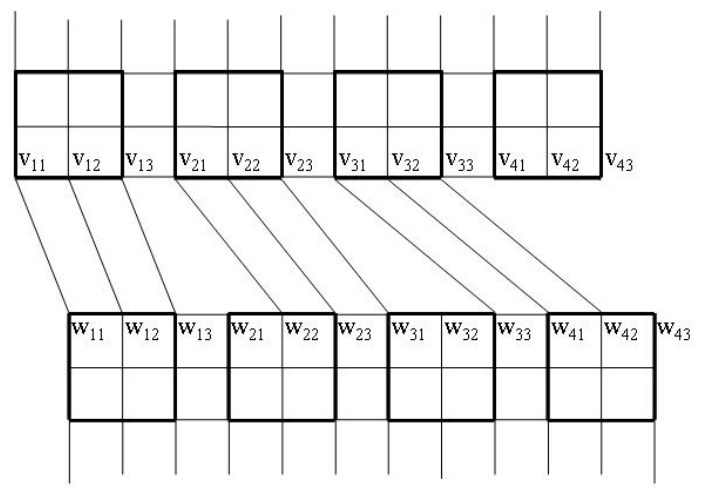

Fig. 3. How to vertically connect two copies of $B$ when $d=2$

of a top-bottom path in this paper as the demonstration of the existence for a right-left path is similar. For each $j \in\left[n_{k}\right]$, we define the set $I_{j}$ as:

$$
I_{j}:=I \cap\{(2 d-1)(j-1)+1,(2 d-1)(j-1)+2, \ldots,(2 d-1) j\}
$$

Since

$$
\left|I_{1}\right|+\cdots+\left|I_{n_{k}}\right|=|I|=t_{k+1}=d^{k+1}-1
$$

and each $\left|I_{j}\right| \leq 2 d-1$, there are at least $\left(n_{k}-t_{k}\right)$ subsets having at most $(d-1)$ elements. Indeed, in the opposite case, we would have:

$$
\left|I_{1}\right|+\cdots+\left|I_{n_{k}}\right| \geq d\left(n_{k}-\left(n_{k}-t_{k}-1\right)\right)=d \cdot d^{k}=d^{k+1},
$$

which would contradict (5). Assume that $S \subseteq\left[n_{k}\right]$ is the set of these indices (i.e. for each $\left.j \in S,\left|I_{j}\right| \leq d-1\right)$. We have: $\left|\left[n_{k}\right] \backslash S\right| \leq t_{k}$. By the induction hypothesis, there is a $\left(\left[n_{k}\right] \backslash S\right)$-avoiding top-bottom path in $\mathcal{G}_{r e c}\left(\ell_{k}\right)$, i.e., the colors used on this path all belong to $S$. Let $v_{1}, \ldots, v_{m}$ be the vertices of the path and denote the color of node $v_{j}$ as $c_{j} \in S(j \in[m])$.

Now, we show there is an $I$-avoiding top-bottom path in $\mathcal{G}_{\text {rec }}\left(\ell_{k+1}\right)$. In $\mathcal{G}_{\text {rec }}\left(\ell_{k+1}\right)$, each node $v_{j}$ has been replaced by a copy $B_{v_{j}}$ with colors in $\left\{(2 d-1)\left(c_{j}-\right.\right.$ $\left.1)+1,(2 d-1)\left(c_{j}-1\right)+2, \ldots,(2 d-1) c_{j}\right\}$. Since the color set $I_{c_{j}}$ satisfies $\left|I_{c_{j}}\right| \leq d-1$, by Proposition 3 we deduce that there are $\mathcal{B}_{d}$ horizontal and $\mathcal{B}_{d}$ vertical $I_{c_{j}}$-avoiding paths in $B_{v_{j}}$.

One can show that this property involves the existence of an $I$-avoiding top-bottom path in $\mathcal{G}_{r e c}\left(\ell_{k+1}\right)$. This top-bottom path is the connection of an $I_{c_{1}}$-avoiding path (from $B_{v_{1}}$ ), an $I_{c_{2}}$-avoiding path (from $B_{v_{2}}$ ), . , an $I_{c_{m}}$-avoiding path (from $B_{v_{m}}$ ). The reader can find more details about this process in Appendix B A similar demonstration leads to the existence of an $I$-avoiding right-left path in $\mathcal{G}_{r e c}\left(\ell_{k+1}\right)$ which achieves the demonstration of our theorem.

The communication complexity of the protocol to $t_{k}$-privately compute the function $f_{G}\left(x_{1}, \ldots, x_{n_{k}}\right)$ using the previous admissible PDAG is $O\left(n_{k} \ell_{k}^{2}\right)$ group elements where 


$$
\ell_{k} \leq \mathcal{B}_{d}^{k}\left(\mathcal{B}_{d}+1\right)^{k-1} \leq 2^{(2 d-1) k} \times 2^{(2 d-1)(k-1)} \leq 2^{2 k(2 d-1)} \leq n_{k}^{\frac{2(2 d-1)}{\log _{2}(2 d-1)}}
$$

Note that the last inequality comes from $2^{k}=n_{k}^{\frac{1}{\log _{2}(2 d-1)}}$.

Now, we generalize our result to any $(t, n)$ where $t=O\left(n^{1-\epsilon}\right)$ for any fixed positive $\epsilon$. The class $O\left(n^{1-\epsilon}\right)$ is the set of all functions $f$ such that: $\exists \tau_{f}>0 \exists n_{0}>0: \forall n \geq$ $n_{0} f(n) \leq \tau_{f} n^{1-\epsilon}$. In our case, the function $f$ is the privacy level $t$. Our main result is stated as follows.

Theorem 4. For any fixed $\epsilon>0$, for any fixed $\tau>0$, there exists a constant $n_{\epsilon, \tau} \in \mathbb{N}$, such that for any $n \geq n_{\epsilon, \tau}$, if $t \leq \tau n^{1-\epsilon}$, then there exists a black-box $t$-private protocol to compute $f_{G}$ with communication complexity polynomial in $n$. Moreover, there is a deterministic polynomial time algorithm to construct the protocol.

Proof. We fix $\epsilon>0$ and $\tau>0$. We set $d=2^{\left\lceil\frac{2}{\epsilon}\right\rceil-1}$ and $k=\left\lfloor\log _{(2 d-1)} n\right\rfloor$. We have $d \geq 2$. If $n \geq 2 d-1$ then $k \geq 1$. In such a condition, we can apply Theorem 3 for the pair $(k, d)$. There exists a $t_{k}$-private protocol to compute the value $f_{G}\left(x_{1}, \ldots, x_{n_{k}}\right)$ using $O\left(n_{k} \ell_{k}^{2}\right)$ group elements where $t_{k}, n_{k}, \ell_{k}$ are defined as in Theorem 3 It is clear that the construction also $t^{\prime}$-privately computes $f_{G}\left(x_{1}, \ldots, x_{n^{\prime}}\right)$ for any $\left(t^{\prime}, n^{\prime}\right)$ such that $t^{\prime} \leq t_{k}$ and $n^{\prime} \geq n_{k}$. So, we only need to show $\tau n^{1-\epsilon} \leq t_{k}, n \geq n_{k}$ and $\ell_{k}=\operatorname{poly}(n)$. Due to our choice of $d$ and $k$, we have:

$$
n_{k} \leq(2 d-1)^{\left\lfloor\log _{(2 d-1)} n\right\rfloor} \leq(2 d-1)^{\log _{(2 d-1)} n} \leq n
$$

And:

$$
t_{k} \geq d^{\left\lfloor\log _{(2 d-1)} n\right\rfloor}-1 \geq d^{\log _{(2 d-1)} n-1}-1 \geq \frac{n^{\frac{\log _{2} d}{\log _{2}(2 d-1)}}}{d}-1 \geq \frac{n^{\frac{\log _{2} d}{\log _{2} 2 d}}}{d}-1
$$

Since $d=2^{\left\lceil\frac{2}{\epsilon}\right\rceil-1}$, we get:

$$
t_{k} \geq \frac{n^{\frac{\left\lceil\frac{2}{\epsilon}\right\rceil-1}{\left\lceil\frac{2}{\epsilon}\right\rceil}}}{2^{\left\lceil\frac{2}{\epsilon}\right\rceil-1}}-1 \geq \frac{n^{1-\frac{\epsilon}{2}}}{2^{\left\lceil\frac{2}{\epsilon}\right\rceil-1}}-1 \geq \frac{n^{\frac{\epsilon}{2}}}{2^{\left\lceil\frac{2}{\epsilon}\right\rceil-1}} n^{1-\epsilon}-1
$$

Since $\epsilon$ is a fixed positive constant, the mapping $n \mapsto \frac{n^{\frac{\epsilon}{2}}}{2^{\left\lceil\frac{2}{\epsilon}\right\rceil-1}}$ has an infinite limit.

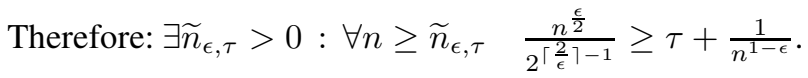

Remember that we early required $n \geq 2 d-1$ in order to use Theorem 3 If we set $n_{\epsilon, \tau}:=\max \left(2 d-1, \widetilde{n}_{\epsilon, \tau}\right)$ then:

$$
\forall n \geq n_{\epsilon, \tau}\left\{\begin{array}{l}
n_{k} \leq n \\
t_{k} \geq \tau n^{1-\epsilon} \geq t
\end{array}\right.
$$

It remains to argue about $\ell_{k}$. Since $n_{k} \leq n$, we have: $\ell_{k} \leq n^{\frac{2(2 d-1)}{\log _{2}(2 d-1)}}$. Since $d$ is independent from $n, \ell_{k}$ is upper bounded by a polynomial in $n$. 
The previous theorem claims that for any fixed $\epsilon$, if $n$ is chosen large enough then we can $t$-privately compute $f_{G}$ for any $t=O\left(n^{1-\epsilon}\right)$. Such an asymptotic survey is also performed in [5]. However, in practical applications, the number of participants is not asymptotically large. The deterministic construction by Desmedt et al. has polynomial cost when $t=O(\log n)$. We now present a result valid for any group size $n$ which guarantees privacy for larger $t$ 's than in [5] using polynomial communication as well.

Theorem 5. For any positive integer n no smaller than 3, there exists a black-box protocol for $f_{G}$ which is $\left(\left\lceil\frac{n^{\log _{3} 2}}{2}\right\rceil-1\right)$-private. It requires the $n$ participants to exchange $O\left(n^{6}\right)$ group elements. Moreover, there is a deterministic polynomial time algorithm to construct the protocol.

Proof. We set $d=2$ and $k:=\left\lfloor\log _{3}(n)\right\rfloor$. The protocol obtained using Theorem 3 has parameter $t_{k} \geq \frac{n^{\log _{3} 2}}{2}-1$ and $n_{k} \leq n$. We have: $\mathcal{B}_{2}=3$. Therefore: $\ell_{k} \leq \frac{n^{1+2} \log _{3} 2}{4}$. Thus, we obtain: $n_{k} \ell_{k}^{2}=O\left(n^{6}\right)$.

\section{Conclusion and Open Problems}

In this paper, we first demonstrated that we could construct a probabilistic $t$-private protocol computing the $n$-product function over any non-Abelian group for any $t$ up to $\frac{n}{2+\epsilon}$ (for any fixed positive $\epsilon$ ), thus nearly matching the known upper bound $\left\lceil\frac{n}{2}\right\rceil-1$. As the communication complexity of our construction is $O\left(n^{3}\right)$ group elements, this result answers one of the questions asked by Desmedt et al. concerning the largest collision resistance achievable with an admissible PDAG of size polynomial in $n$. Note that Desmedt et al. indicated the discovery of a construction for $(n, t)=(24,11)$ improving locally their own theoretical bound $\frac{n}{2.948}$ since $11 \approx \frac{24}{2.182}$. Our result demonstrates the existence of such a construction for any fixed positive $\epsilon$ (in [5], we have the particular case $\epsilon=0.182$ ). Since the scheme developed in [5] (exclusively valid for $t<\frac{n}{2.948}$ ) only requires $O\left(n t^{2}\right)$ elements to be exchanged, a direction to further investigate is the existence of a (randomized) $t$-private protocol for any $t \leq\left\lceil\frac{n}{2}\right\rceil-1$ having at most the cost of Desmedt et al.'s scheme.

Second, we showed that it was possible to construct a deterministic $t$-private $n$-party protocol to compute $f_{G}$ having a polynomial communication cost for any $t=O\left(n^{1-\epsilon}\right)$. For practical purpose, one may want to optimize the choice of parameters in our construction. For example, we have proved that one could $t$-privately compute $f_{G}$ for any $(t, n)$ satisfying $t \leq\left\lceil\frac{n^{\log _{3} 2}}{2}\right\rceil-1$.

Desmedt et al. argued that the reduction from a protocol computing the $n$-product to a subroutine computing the shared 2-product extended to the more general function $\widetilde{f}_{G}\left(x_{1}, \ldots, x_{m}\right):=x_{1} \cdot x_{2} \cdot \ldots \cdot x_{m}$ where $m \geq n$ and each of the $n$ parties holds one or more input values. This ensured the validity of their protocol to securely compute $\widetilde{f}_{G}$ as well. Since the constructions that we presented are particular admissible PDAGs, our results are also valid to compute $\widetilde{f}_{G}$.

Our work leads to the following two questions. First, is it possible to reduce the communication cost when $t=O\left(n^{1-\epsilon}\right)$ ? Second, can we generalize this approach to 
design a deterministic polynomial communication cost algorithm for any $t$ up to the threshold $\left\lceil\frac{n}{2}\right\rceil-1$ ?

Apart from the previous points which constitute directions to improve the security for the passive adversary model, a problem which requires attention is the possibility of achieving secure computation of $f_{G}$ against malicious parties. Indeed, even if multiparty computation can be used with small groups (as in the case of the Millionaires' problem [19]), the general purpose is to enable large communication groups to perform common computations and the larger the number of parties is, the more likely (at least) one of them will deviate from the given protocol.

\section{Acknowledgments}

The authors are grateful to the anonymous reviewers for their comments to improve the quality of this paper. The three authors' work was supported in part by the National Natural Science Foundation of China grant 60553001 and the National Basic Research Program of China grants 2007CB807900 and 2007CB807901. Xiaoming Sun's research was also funded by the National Natural Science Foundation of China under grant 60603005 . Christophe Tartary's work was also financed by the Ministry of Education of Singapore under grant T206B2204.

\section{References}

[1] Ben-Or, M., Goldwasser, S., Wigderson, A.: Completeness theorems for non-cryptographic fault-tolerant distributed computation. In: 20th Annual ACM Symposium on Theory of Computing, Chicago, USA, pp. 1-10. ACM Press, New York (1988)

[2] Bollobàs, B., Riordan, O.: Percolation. Cambridge University Press, Cambridge (September 2006)

[3] Cramer, R., Damgård, I.B., Maurer, U.: General secure multi-party computation from any linear secret-sharing scheme. In: Preneel, B. (ed.) EUROCRYPT 2000. LNCS, vol. 1807, pp. 316-334. Springer, Heidelberg (2000)

[4] Damgård, I.B., Ishai, Y.: Scalable secure multiparty computation. In: Dwork, C. (ed.) CRYPTO 2006. LNCS, vol. 4117, pp. 501-520. Springer, Heidelberg (2006)

[5] Desmedt, Y., Pieprzyk, J., Steinfeld, R., Wang, H.: On secure multi-party computation in black-box groups. In: Menezes, A. (ed.) CRYPTO 2007. LNCS, vol. 4622, pp. 591-612. Springer, Heidelberg (2007)

[6] Diffie, W., Hellman, M.E.: New directions in cryptography. IEEE Transactions on Information Theory 22(6), 644-654 (1976)

[7] El Gamal, T.: A public-key cryptosystem and a signature scheme based on discrete logarithms. IEEE Transactions on Information Theory 31(4), 469-472 (1985)

[8] Goldreich, O., Vainish, R.: How to solve any protocol problem - an efficiency improvement. In: Pomerance, C. (ed.) CRYPTO 1987. LNCS, vol. 293, pp. 73-86. Springer, Heidelberg (1988)

[9] Hammersley, J.M.: Percolation processes: Lower bounds for the critical probability. The Annals of Mathematical Statistics 28(3), 790-795 (1957)

[10] Hirt, M., Maurer, U.: Robustness for free in unconditional multi-party computation. In: Kilian, J. (ed.) CRYPTO 2001. LNCS, vol. 2139, pp. 101-118. Springer, Heidelberg (2001) 
[11] Hirt, M., Maurer, U., Przydatek, B.: Efficient secure multi-party computation. In: Okamoto, T. (ed.) ASIACRYPT 2000. LNCS, vol. 1976, pp. 143-161. Springer, Heidelberg (2000)

[12] Hirt, M., Nielsen, J.B.: Robust multiparty computation with linear communication complexity. In: Dwork, C. (ed.) CRYPTO 2006. LNCS, vol. 4117, pp. 463-482. Springer, Heidelberg (2006)

[13] Kesten, H.: Percolation Theory for Mathematicians. Birkhäuser, Basel (November 1982)

[14] Lang, S.: Algebra (Revised Third Edition). Springer, Heidelberg (November 2002)

[15] Magliveras, S.S., Stinson, D.R., van Trung, T.: New approaches to designing public key cryptosystems using one-way functions and trapdoors in finite groups. Journal of Cryptology 15(4), 285-297 (2002)

[16] Paeng, S.-H., Ha, K.-C., Kim, J.H., Chee, S., Park, C.: New public key cryptosystem using finite non Abelian groups. In: Kilian, J. (ed.) CRYPTO 2001. LNCS, vol. 2139, pp. 470485. Springer, Heidelberg (2001)

[17] Rivest, R.L., Shamir, A., Adleman, L.M.: A method for obtaining digital signatures and public key cryptosystems. Communication of the ACM 21(2), 120-126 (1978)

[18] Shor, P.W.: Polynomial-time algorithms for prime factorization and discrete logarithms on a quantum computer. SIAM Journal on Computing 26(5), 1484-1509 (1997)

[19] Yao, A.C.-C.: Protocols for secure computations. In: 23rd Annual IEEE Symposium on Foundations of Computer Science, Chicago, USA, November 1982, pp. 80-91. IEEE Press, Los Alamitos (1982)

\section{A Proof of Proposition 3}

Let $I$ be a $(d-1)$-color subset of $[2 d-1]$. In [5], Desmedt et al. demonstrated that there were a $I$-avoiding top-bottom path and a $I$-avoiding right-left path in $\mathcal{G}_{\text {tri }}\left(\ell_{1}, \ell_{1}\right)$. They also showed that those two paths were straight lines. Thus, one can remove the diagonal edges of $\mathcal{G}_{\text {tri }}\left(\ell_{1}, \ell_{1}\right)$ while preserving those paths. This means that there exist a $I$-avoiding top-bottom path and a $I$-avoiding right-left path in $\mathcal{G}_{r e c}\left(\ell_{1}\right)$ which are straight lines.

Since $B$ is a- $\left(\mathcal{B}_{d}+1\right) \times\left(\mathcal{B}_{d}+1\right)$-copy of $\mathcal{G}_{\text {rec }}\left(\ell_{1}\right)$ and, due to the vertical/horizontal connections of these copies, we deduce that there are $\left(\mathcal{B}_{d}+1\right) I$-avoiding top-bottom paths and $\left(\mathcal{B}_{d}+1\right) I$-avoiding right-left paths in $B$. Moreover, each of these paths is a straight line.

\section{B Connection of Color Avoiding Paths}

It was shown in the proof of Theorem 3 that each block $B_{c_{i}}$ had $\mathcal{B}_{d}$ horizontal and $\mathcal{B}_{d}$ vertical $I_{c_{i}}$-avoiding paths. In this appendix, we show how to construct a $I$-avoiding top-bottom path in $\mathcal{G}_{r e c}\left(\ell_{k+1}\right)$. Our path will start at the top of $B_{v_{1}}$ and ends at the bottom of $B_{v_{m}}$.

Every grid from the family $\left(\mathcal{G}_{\text {rec }}\left(\ell_{\lambda}\right)\right)_{\lambda>1}$ is a square grid. Thus, the sequence of blocks $B_{v_{1}}, \ldots, B_{v_{m}}$ in $\mathcal{G}_{r e c}\left(\ell_{k+1}\right)$ is determined by the position of $B_{v_{1}}$ as well as the $m$-tuple of letters from $\{\mathfrak{L}, \mathfrak{R}, \mathfrak{T}, \mathfrak{B}\}$ (Left, $\mathfrak{R i g h t}, \mathfrak{T}$ op, $\mathfrak{B}$ ottom) indicating the output side of the block $B_{v_{i}}$ for $i \in[m]$. Note that the last letter of the tuple is always $\mathfrak{B}$ since the $I$-avoiding top-bottom path ends at the bottom of $B_{v_{m}}$.

This tuple has the property the two consecutive letters cannot be opposite to each other (i.e, one cannot have $(\mathfrak{L}, \mathfrak{R}),(\mathfrak{R}, \mathfrak{L}),(\mathfrak{T}, \mathfrak{B})$ or $(\mathfrak{B}, \mathfrak{T}))$. This means that you leave 
a block on a different side that you entered it. The reader can check the correctness of this claim by a simple recursive process on the parameter $k$. This property is trivially true for $k=1$ since $\mathcal{G}_{\text {rec }}\left(\ell_{1}\right)=\mathcal{G}_{\text {tri }}\left(\ell_{1}\right)$. The recursion follows from the path construction that we will design below.

Proposition 4. Let $i$ be any element of $[m]$. Assume that $\mathcal{N}$ is any node on a side of $B_{v_{i}}$ belonging to a $I_{c_{i}}$-avoiding straight line path. For each other side $\mathfrak{S}_{i}$ of $B_{v_{i}}$, we can construct a $I_{c_{i}}$-avoiding path from $\mathcal{N}$ to any of the $\left(\mathcal{B}_{d}+1\right)$ nodes on $\mathfrak{S}_{i}$ belonging to a $I_{c_{i}}$-avoiding straight line path.

Proof. We only provide a proof when $\mathcal{N}$ is on the top side of $B_{v_{i}}$ (the three other cases are similar). The three possible output sides are $\mathfrak{B}, \mathfrak{L}$ and $\mathfrak{R}$. The block $B_{v_{i}}$ is a- $\left(\mathcal{B}_{d}+1\right) \times\left(\mathcal{B}_{d}+1\right)$-copy of the original grid $\mathcal{G}_{\text {rec }}\left(\ell_{1}\right)$. Thus, $B_{v_{i}}$ can be treated as a $\left(\mathcal{B}_{d}+1\right) \times\left(\mathcal{B}_{d}+1\right)$ array of grids $\mathcal{G}_{\text {rec }}\left(\ell_{1}\right)$. Based on this observation, we will use the terminology grid-row (respectively grid-column) to denote a set of $\mathcal{B}_{d}+1$ horizontal (respectively vertical) grids $\mathcal{G}_{r e c}\left(\ell_{1}\right)$ in $B_{v_{i}}$.

1. $\mathfrak{S}_{i}=\mathfrak{B}$. The vertical $I_{c_{i}}$-avoiding path starting at node $\mathcal{N}$ intersects the horizontal $I_{c_{i}}$-avoiding path located within the bottom grid-row of $B_{v_{i}}$ at node $\mathcal{I}$. That horizontal path intersects each of the $\mathcal{B}_{d}+1$ vertical $I_{c_{i}}$-avoiding paths (one within each grid-column) at $\mathcal{I}_{1}, \ldots, \mathcal{I}_{\mathcal{B}_{d}+1}$. Note that $\mathcal{I}=\mathcal{I}_{\mu}$ for some $\mu \in\left[\mathcal{B}_{d}+1\right]$. Once we are at one of the $\mathcal{I}_{j}$ 's, we simply go vertically downwards to the node $\mathcal{N}_{j}^{\prime}$ located at the bottom side of the block $B_{v_{i}}$.

Thus, we can construct a path from $\mathcal{N}$ to each of the $\mathcal{B}_{d}+1$ output nodes on the bottom side of $B_{v_{j}}$ belonging to the vertical $I_{c_{i}}$-avoiding paths. Those paths are $\left(\mathcal{N}, \mathcal{I}, \mathcal{I}_{j}, \mathcal{N}_{j}^{\prime}\right)$ for $j \in\left[\mathcal{B}_{d}+1\right]$.

2. $\mathfrak{S}_{i}=\mathfrak{R}$. The vertical $I_{c_{i}}$-avoiding path starting at node $\mathcal{N}$ intersects the horizontal $I_{c_{i}}$-avoiding path located within the top grid-row of $B_{v_{i}}$ at node $\mathcal{I}$. That horizontal path intersects the vertical $I_{c_{i}}$-avoiding path located within the rightmost grid-column of $B_{v_{i}}$ at node $\widetilde{\mathcal{I}}$. This vertical path intersects each of the $\mathcal{B}_{d}+1$ horizontal $I_{c_{i}}$-avoiding paths (one within each grid-row) at $\widetilde{\mathcal{I}}_{1}, \ldots, \widetilde{\mathcal{I}}_{\mathcal{B}_{d}+1}$. As before, we get: $\widetilde{\mathcal{I}}=\widetilde{\mathcal{I}}_{\mu}$ for some $\mu \in\left[\mathcal{B}_{d}+1\right]$. Once we are at one of the $\widetilde{\mathcal{I}}_{j}$ 's, we horizontally go rightwards to the node $\mathcal{N}_{j}^{\prime}$ located on the right hand side of the block $B_{v_{i}}$.

Thus, we can construct a path from $\mathcal{N}$ to each of the $\mathcal{B}_{d}+1$ output nodes on the right hand side of $B_{v_{j}}$ belonging to the horizontal $I_{c_{i}}$-avoiding paths. Those paths are $\left(\mathcal{N}, \mathcal{I}, \widetilde{\mathcal{I}}, \widetilde{\mathcal{I}}_{j}, \mathcal{N}_{j}^{\prime}\right)$ for $j \in\left[\mathcal{B}_{d}+1\right]$.

3. $\mathfrak{S}_{i}=\mathfrak{L}$. This is analogous to the previous case.

We can finally construct a $I$-avoiding top-bottom path in $\mathcal{G}_{r e c}\left(\ell_{k+1}\right)$. We denote the $m$-tuple of output sides as $\left(\mathfrak{S}_{1}, \ldots, \mathfrak{S}_{m}\right)$. As previously said, we have: $\mathfrak{S}_{m}=\mathfrak{B}$.

We start at any node $N_{1}$ located on the top side of $B_{v_{1}}$ and on a vertical $I_{c_{1}}$-avoiding path. Using Proposition 4, we can connect $N_{1}$ to any of the $\mathcal{B}_{d}+1$ nodes on side $\mathfrak{S}_{1}$ of $B_{v_{1}}$ using a $I_{c_{1}}$-avoiding path. An important remark is that each block of the whole grid $\mathcal{G}_{r e c}\left(\ell_{k+1}\right)$ is a set of $\left(\mathcal{B}_{d}+1\right) \times\left(\mathcal{B}_{d}+1\right)$ identical copies of $\mathcal{G}_{\text {rec }}\left(\ell_{1}\right)$ (including the coloring). As a consequence, these $\mathcal{B}_{d}+1$ nodes have the same location in their respective copies of $\mathcal{G}_{\text {rec }}\left(\ell_{1}\right)$. Given the connection process between any pair of blocks 
within $\mathcal{G}_{\text {rec }}\left(\ell_{k+1}\right)$, one of these $\mathcal{B}_{d}+1$ nodes must be connected to a node $N_{2}$ from block $B_{v_{2}}$ belonging to a $I_{c_{2}}$-avoiding straight line path. Similarly, $N_{2}$ is connected via a $I_{c_{2}}$-avoiding path in $B_{v_{2}}$ to a node $N_{3}$ from $B_{v_{3}}$ belonging to a $I_{c_{3}}$-avoiding straight line path. If we repeat this process for each of the remaining blocks, we obtain a set of $m-1$ nodes $N_{1}, \ldots, N_{m-1}$. The last node $N_{m-1}$ can be connected to a node $N_{m}$ on the bottom side of $B_{v_{m}}$ using a $I_{c_{m}}$-avoiding path. Thus, $N_{1}$ (top side of $\mathcal{G}_{\text {rec }}\left(\ell_{k+1}\right)$ ) is connected to $N_{m}$ (bottom side of $\mathcal{G}_{\text {rec }}\left(\ell_{k+1}\right)$ ) using a $I$-avoiding path which achieves the demonstration of our theorem.

Remark: As claimed above, this construction involves that the two consecutive side letters of the $m$-tuple cannot be opposite to each other. 